\title{
Miranda
}

Revue pluridisciplinaire du monde anglophone /

Multidisciplinary peer-reviewed journal on the English-

speaking world

17 | 2018

Paysages et héritages de David Bowie

\section{Ryan Heffington et Kenzo World. La danse au service du marketing ou le marketing au service de la danse?}

\section{Clément Djaaï--Mourgues}

\section{(2) OpenEdition}

Journals

Édition électronique

URL : http://journals.openedition.org/miranda/13687

DOI : $10.4000 /$ miranda. 13687

ISSN : 2108-6559

\section{Éditeur}

Université Toulouse - Jean Jaurès

\section{Référence électronique}

Clément Djaaï--Mourgues, « Ryan Heffington et Kenzo World. La danse au service du marketing ou le marketing au service de la danse ? », Miranda [En ligne], 17 | 2018, mis en ligne le 28 septembre 2018, consulté le 16 février 2021. URL : http://journals.openedition.org/miranda/13687 ; DOI : https:// doi.org/10.4000/miranda.13687

Ce document a été généré automatiquement le 16 février 2021.

\section{cc)}

Miranda is licensed under a Creative Commons Attribution-NonCommercial-NoDerivatives 4.0 International License. 


\title{
Ryan Heffington et Kenzo World. La danse au service du marketing ou le marketing au service de la danse?
}

\author{
Clément Djaaï--Mourgues
}

1 Ryan Heffington, Margaret Qualley, Maddie Ziegler, ces noms ne vous sont peut-être pas familiers; pourtant, il ne fait aucun doute que, durant ces dernières années, vous vous êtes nécessairement retrouvés confrontés à leur travail au moins une fois. Et pour cause, ils sont les acteurs majeurs de la récente popularisation de la danse contemporaine. À présent, si j'évoque le clip de 'Chandelier' (2014) de Sia, celui de 'Cheap Thrills' (2016) ou encore la publicité Kenzo World, tout devient certainement plus clair. Rien d'étonnant à cela. Ces vidéos ont eu un effet viral et ont certainement dû vous marquer par leur originalité, et ce que vous soyez amateur de danse ou non.

2 C'est pourtant au milieu du $20^{\mathrm{e}}$ siècle que remontent les origines de la danse contemporaine. Toutefois, il est vrai que si la danse, sous diverses formes, et au même titre que la musique, est un art qui fait partie de la vie de tous les jours de tout un chacun de manière plus ou moins consciente, sa dérivée dite contemporaine souffre encore d'une transition vers une culture plus populaire (ou mainstream) qui ne semble pas avoir eu lieu. Cette forme de danse porte encore de nos jours l'étiquette d'un art plus complexe, voire élitiste, qu'à l'instar d'un bon vin il faut être initié pour apprécier pleinement. En 2014, la collaboration entre le chorégraphe américain Ryan Heffington, la chanteuse Sia et la jeune danseuse Maddie Ziegler pour le clip de 'Chandelier', totalisant près de 2 milliards de vues sur YouTube, a un effet viral sur la toile et le chorégraphe reçoit le MTV Video Music Award de la meilleure chorégraphie. Depuis, et notamment grâce à son travail avec Sia, il semble que le chorégraphe participe à une vulgarisation de la danse contemporaine, vulgarisation étant à entendre ici dans son sens étymologique, à savoir une mise à disposition de cet art au plus grand nombre. L'utilisation ici d'un terme galvaudé tel que celui de vulgarisation n'est pas gratuite. En effet, s'il existe deux termes galvaudés de nos jours, ce sont bien ceux de 'commercial' et de 'populaire'. Ces deux termes ont eu tendance à être amalgamés dans un 
néologisme dont le sens se rapproche plutôt du populaire et la terminologie retenue a été celle de 'commercial', le tout portant une connotation négative. On dit donc aujourd'hui qu'une chanson est 'commerciale' si elle rencontre un franc succès auprès du grand public. Du côté du septième art, on oppose le film mainstream ou blockbuster au film dit 'd'auteur'. Les théoriciens de la danse contemporaine, de leur côté, sont tiraillés. D'un côté, ils déplorent le manque de popularité de cette danse auprès du grand public. D'un autre, pourtant, ils ne semblent pas vouloir confier cet art aux yeux de spectateurs qui n'auraient pas toutes les armes pour le comprendre. Patrick Germain-Thomas et Dominique Pagès ont écrit :

La danse contemporaine est peu présente dans les médias grand public et son image est trouble et dispersée. Elle est souvent faussée par une imagerie publicitaire séduisante qui se focalise sur l'esthétique spectrale de corps dansants élégants et enchanteurs. La grâce du geste et la splendide énigme des postures prennent le pas sur l'expérience intime du mouvement. Dans une société où le modèle du corps sportif et performatif occupe une place prédominante, les corps dansants en viennent à être emblématisés, notamment à travers des situations de tension paroxystiques. Cela va au détriment de la singularité des engagements de la danse contemporaine qui privilégie historiquement la justesse et la richesse expressive du mouvement.

Néanmoins, le clip de 'Chandelier', à l'époque, avait conquis à la fois le public mais aussi les grands pontes du journalisme culturel. C'est donc certainement fort de ce succès que le chorégraphe Ryan Heffington, en 2016, s'est lancé dans le pari risqué de chorégraphier un clip aux fins ouvertement commerciales, à savoir la publicité du dernier né de la parfumerie Kenzo: Kenzo World. Et ce n'est pas d'une équipe de débutants dont la maison Kenzo s'est entourée pour promouvoir son parfum. En effet, aux côtés du chorégraphe, la présence derrière la caméra du réalisateur américain multi-oscarisé Spike Jonze (Her (2013), Dans la Peau de John Malkovich (1999)) laissait espérer un clip aux allures de court-métrage. Enfin, c'est à la jeune ballerine et actrice Margaret Qualley, moins connue du grand public mais au talent très prometteur, que le chorégraphe a fait confiance pour exécuter sa chorégraphie.

4 Ainsi, dans le contexte expliqué précédemment contrebalancé par la présence d'une équipe très prometteuse aux commandes du projet, je me suis posé la question suivante : ce clip, à la portée commerciale non dissimulée, en perd-il pour autant sa valeur artistique ? C'est ce que nous allons tenter de décrypter.

\section{Ryan Heffington : chorégraphe et auteur}

5 Si la danse, du côté du spectateur, est un art qui se regarde ou se vit comme une expérience kinesthésique, de celui du chorégraphe, elle est également un art qui s'écrit, possédant son propre système de notation aussi élaboré que le sont les mots en littérature et les notes en musique. En ce sens, le travail du chorégraphe se rapproche déjà de celui de l'auteur. Mais un auteur se caractérise également par des thématiques qui lui sont propres permettant d'établir des ponts entre chacune de ses œuvres. Influencé par les chorégraphes contemporains de la première heure tels que Marta Graham, dont on retrouve l'aspect psychanalytique mais aussi postmoderne dans ses œuvres comme le montrera cette analyse, Ryan Heffington avait déjà dégagé, dans ses clips musicaux pour Sia, certaines thématiques, comme celle du cloisonnement, qu'il soit physique ou psychologique. Selon la chanteuse, c'est un personnage "gauche », 
«primitif» et «au bord de la crise de nerfs» (Kourlas 8) car séquestré que mettait en mouvement le chorégraphe dans le clip de sa chanson 'Chandelier'. Dans la publicité Kenzo World, il est question d'une jeune femme dont la liberté de s'exprimer est bridée par la société du paraître. En ce sens, il est d'ailleurs intéressant de remarquer que Sia, avec qui Heffington collabore régulièrement, est une artiste qui cache son visage du public et chante « there's a scream inside that we all try to hide » (« il y a un cri en nous que l'on essaye tous de cacher ») ("Bird Set Free », 2015). Une autre thématique reliant les deux artistes est un refus de montrer le beau conventionnel, privilégiant plutôt le bizarre, l'adjectif anglais freaky étant certainement le plus juste ici. Ainsi, dans les chorégraphies de Ryan Heffington tant que sur les pochettes d'albums de Sia, on peut voir des grimaces, des poils sous les bras, des corps déformés, en bref tout ce qui tend à s'éloigner de ce qu'est la beauté normée dans la société actuelle.

6 Avant de s'intéresser à la manière dont Ryan Heffington a développé ses thématiques dans la publicité Kenzo World, il convient de faire le point sur la ligne artistique de Kenzo, afin de mettre en parallèle ces deux éléments.

\section{Kenzo et l'évasion}

7 Kenzo est d'abord une marque de vêtements, qui a su se démarquer des autres maisons de luxe en prenant la voie du streetwear, notamment ces dernières années, en proposant des couleurs vives voire flashy, des motifs imposants et en choisissant des symboles tels que le tigre, un animal inspirant un esprit sauvage, mais invitant également au voyage de par son origine asiatique, et dont la représentation sur les vêtements Kenzo rappelle les motifs des souvenirs jackets, un vêtement datant de la Seconde Guerre mondiale, originellement confectionné à partir des chutes de toiles de parachutes et brodé par la suite, que les soldats ainsi que les touristes rapportaient à leur famille du Japon. Sur le plan artistique, la marque exprime donc un réel souhait de s'affranchir des conventions du luxe en proposant des vêtements non pas discrets et distingués mais plutôt des vêtements de caractère, permettant d'exprimer son caractère, aux couleurs obligeant à s'assumer et s'amuser du regard que les autres pourraient porter sur soi, mais également des conventions du genre avec une collection femme proche de la collection homme pour ne pas dire totalement androgyne. Enfin, la maison Kenzo souhaite inviter ses clients à découvrir le monde de leurs propres yeux, l'œil étant également l'un de ses motifs emblématiques, cela expliquant peut-être le choix du nom de son nouveau parfum, Kenzo World, un parfum présenté par la marque de la manière suivante :

Les directeurs artistiques Carol Lim et Humberto Leon retranscrivent leur vision de la mode et créent leur premier parfum. Un monde à leur image, audacieux, spontané, surprenant et coloré. Un monde qui prône la liberté d'expression, la diversité et la créativité. Un parfum dynamique et joyeux, une nouvelle dimension de l'esprit KENZO. (« Kenzo World »)

\section{L'anti-publicité pour parfum}

Dans l'imaginaire collectif, la publicité de parfum est extrêmement stéréotypée. On imagine directement une femme sublime, dans une robe longue, arborant une parure de bijoux plus scintillants les uns que les autres et descendant l'escalier central d'un palais luxueux tandis que les convives admirent sa beauté. 
9 Ici, le cadre est une salle de réception, pas n'importe laquelle d'ailleurs, celle du Pavillon Dorothy Chandler de Los Angeles, devenu un emblème mythique du rassemblement protocolaire pour avoir accueilli la cérémonie des Oscars à 19 reprises. Un homme est en train de prononcer un discours de remerciement, le genre de discours réputé pour être interminable, et dont les blagues doivent faire sourire poliment au moment où le locuteur l'a décidé mais ne font jamais rire. Au milieu de cette assemblée, tout de noir et de blanc vêtue, une jeune femme, légèrement décoiffée et portant une robe verte, ne rit pas. Elle peine à forcer le sourire et acquiescer semble être son seul moyen de s'exprimer. Dès le départ, les codes de la publicité de parfum classique sont bouleversés. La jeune femme n'est pas le centre d'attention, mais fait au contraire partie de la foule, de ceux qui regardent. Elle sort de la salle, ses larmes coulent, elle regarde le spectateur droit dans les yeux et commence soudain à se plonger dans un état de transe.

10 Le spectateur est témoin de la désarticulation progressive de la danseuse. C'est la lente reprise de contrôle du ça sur le surmoi. Ce sont tout d'abord ses yeux qui se désarticulent, puis ses paupières. Suivent la mâchoire, le cou, les épaules, puis les bras, les hanches, et enfin les jambes. Son corps semble tomber dans un état d'abandon total, dont le cri est certainement la concrétisation ultime. Cette désarticulation du corps s'accompagne d'un rejet des conventions sociales, un besoin de couper la parole ou même de mettre fin aux discours interminables et inintéressants, que la danseuse exprime en mordant sa main mimant un homme en train de parler, mais aussi de renouer avec ses instincts primaires en imitant le singe devant le miroir. Son comportement contraste avec celui des convives présents dans la salle de réception au début du clip, symbolisés par la statue d'un homme, immobile et dénué de tout sentiment, que ni l'énervement, ni la violence, ni même les avances de la jeune femme ne semblent pouvoir perturber. Mais l'aspect destructeur d'un naturel trop longtemps contenu est également montré, tout d'abord au travers de pulsions assassines, puis de manière exacerbée par les pouvoirs destructeurs que la jeune femme développe et utilise contre son environnement.

11 C'est pourquoi le conscient de la danseuse tente à plusieurs reprises de reprendre le dessus, tout d'abord lorsqu'elle défile tel un mannequin à l'étage du prestigieux pavillon avec toute la rigueur et la rigidité qu'il est demandé aux modèles de respecter, mais ce défilé est entrecoupé de mouvements de combat empruntés au kata japonais. Le kata étant une forme de combat qui se pratique généralement seul, le spectateur peut imaginer ici qu'une part de la danseuse lutte contre une autre part d'elle-même. Une fois de plus, c'est de la lutte du surmoi contre le ça, ou inversement, dont il est question. Cette même lutte est observable dans la salle tapissée, où la danseuse peine à retenir ses bras et ses jambes, incontrôlables et imprévisibles. Enfin, lorsqu'elle entre sur scène, Margaret Qualley semble s'efforcer de pratiquer des arabesques, mouvements emblématiques de la danse classique, tandis que son corps la ramène à une danse plus contemporaine. C'est d'ailleurs celui-ci qui prend le dessus au final lorsque la danseuse se jette dans le public à la manière d'une rock star. C'est à une réflexion sur l'évolution de la danse que Ryan Heffington invite le spectateur ici. En effet, tout comme les rock stars se sont affranchies du quatrième mur séparant traditionnellement l'artiste du public et n'étant, au final, qu'une convention dont la raison d'être est plus coutumière que logique, la danse contemporaine est venue bousculer et désaxer des mouvements trop codifiés. Cette idée est très justement 
identifiée par Betty Mercier-Lefèvre, qui, reprenant un concept bourdieusien pour mettre l'accent sur la postmodernité de la danse contemporaine, écrit qu'elle «transgress[e] nos « habitus » chorégraphiques » (Mercier-Lefèvre).

12 L'aspect subversif de ce clip ne concerne pas seulement la danse. Il semble que Ryan Heffington et Spike Jonze ont travaillé main dans la main pour faire de ce spot une antipublicité pour parfum. Si j'ironisais précédemment à propos de la traditionnelle scène des escaliers, c'est parce qu'elle a été détournée dans cette publicité. Ici, la jeune femme ne descend pas lentement des escaliers, elle les remonte à vive allure. Elle n'entre pas dans le champ mais en sort, la multiplication des miroirs environnants empêchant toute possibilité de scopophilie et donnant l'impression que la danseuse quitte le cadre par ses quatre angles. Grâce à la maestria de Spike Jonze, le spectateur ne sait plus où donner du regard et se focalise sur la personnalité morcelée du personnage plutôt que sur sa beauté.

13 Même l'aspect extrêmement genré des publicités de parfum classiques est pris à contre-pied. Margaret Qualley se laisse aller à des mouvements tels que frapper sa poitrine, à en faire douter Tarzan de sa propre virilité, ou à des poses de culturiste.

14 La scène finale, contemplative et filmée au ralenti, lors de laquelle la jeune femme transperce littéralement un œil, est la représentation métaphorique de cette invitation à s'affranchir des codes de la bienséance, de la danse ou encore du genre, dont la peur du regard et du jugement des autres est le cerbère.

15 Pour composer la musique de son court-métrage, le réalisateur Spike Jonze a fait appel à son frère le DJ Sam Spiegel, qui s'est entouré pour l'occasion de l'artiste dancehall Ape Drums ainsi que du chanteur reggae Assassin. Bien que proposant des sonorités plus actuelles, le morceau «Mutant Brain» (2016) composé en collaboration par les trois artistes, en mêlant influences occidentales et africaines, invite à se souvenir des fondements de la danse contemporaine qui fut elle aussi le fruit de la rencontre entre ces deux cultures. Un morceau extrêmement saccadé, dont chacune des pulsations est marquée par un mouvement de la danseuse, donnant à l'ensemble du clip une atmosphère ethnique. C'est certainement au travers de la musique que l'invitation au voyage décrite précédemment, et probablement attendue par la marque pour promouvoir un parfum au nom de World, est la plus présente.

16 C'est véritablement sa propre définition de la danse, et en particulier de la danse contemporaine, celle qu'il affectionne, que nous livre Ryan Heffington à travers cette chorégraphie. Le plus réussi dans ce clip est peut-être la manière dont il a su dissimuler un immense travail de création derrière une volonté de donner l'impression, que chacun a du ressentir au premier visionnage, que Margaret Qualley gesticule de manière totalement aléatoire. Ainsi, l'étrange impression de beauté qui ressort de l'œuvre dans son ensemble semble être inexplicable. La danse contemporaine est, pour Ryan Heffington, l'expression d'un besoin qu'a l'être humain, quel que soit son physique ou son milieu, de renouer avec sa propre corporalité et de cesser de contenir son corps en permanence pour plaire à l'autre ou anticiper le jugement désagréable qu'il pourrait porter sur soi. Ce clip est une invitation à l'évasion, qu'elle soit physique à l'image de la jeune femme qui s'éloigne d'une foule qui l'oppresse, ou intérieure.

17 Le chorégraphe a su utiliser le format qui lui était proposé, à savoir une publicité, et se l'approprier afin d'y développer ses thématiques. C'est avec une grande intelligence qu'il a su utiliser la vitrine qui lui était offerte par Kenzo pour faire connaitre son art et universaliser son message sans céder à la facilité de tomber dans les clichés du genre. 
Finalement, même le rapport au matériel, au parfum en tant que produit, n'est pas inintéressant. Du côté de l'artiste, il symbolise le fait qu'une telle libération est possible, qu'elle n'est pas totalement utopique, mais qu'il ne tient qu'à chacun de la toucher du doigt. Et, bien évidemment, du côté de la marque, il sert à rappeler qu'une telle libération est disponible dans toute bonne parfumerie au prix de 54,99€ les 30 millilitres.

Spike Jonze, le réalisateur, dont le rôle dans la réussite du projet ne doit bien évidemment pas être mis de côté indépendamment du fait que c'est de danse dont il a majoritairement été question dans ce dossier, a également pu développer ses thématiques habituelles dans son court-métrage. La difficulté des relations humaines menant à un détachement total de la réalité est un sujet que le réalisateur affectionne tout particulièrement. Et si ce n'est pas déjà fait, le visionnage de Her vous donnera à la fois l'occasion de découvrir un grand film et de vous en rendre compte.

$\mathrm{Au}$ final, le pari semble réussi pour tous les artistes ayant collaboré dans ce projet. À présent, faut-il boycotter une œuvre d'art sous prétexte qu'elle a des fins mercantiles? C'est à vous d'en juger. En effet, ces deux notions s'opposent souvent dans la mesure où l'art peut être vu comme un refuge, un moyen de rêver, de s'évader, dans une société où tout s'achète. Il nous est tous arrivé de nous laisser emporter, dans une salle de cinéma, par ce que l'on croyait être un court-métrage, ou même le début du film, avant qu'un pack-shot pour un yaourt $0 \%$ nous ramène brutalement à la réalité. Mais l'exemple de Kenzo World nous montre que derrière une publicité se cache souvent un voire plusieurs artistes, tout comme ce sont des artistes qui sont à l'origine d'un parfum ou d'un beau vêtement. Ainsi, on peut tout simplement imaginer dans ce cas qu'un art est venu en sublimer un autre, et que l'on paiera pour acquérir ce parfum tout comme l'on paie une place de cinéma, un ticket de concert, une certaine somme pour aller admirer un tableau dans un musée ou même une fortune pour en faire l'acquisition. Mais ne soyons pas fatalistes, et réjouissons-nous plutôt que la danse contemporaine bénéficie d'une vitrine bien méritée permettant de faire découvrir cet art au plus grand nombre et que l'œuvre de Ryan Heffington, Spike Jonze et la maison Kenzo soit disponible sur tout bon téléviseur, pendant la page de réclame de notre programme favori, et ce gratuitement.

\section{BIBLIOGRAPHIE}

\section{Bibliographie}

Germain-Thomas, Patrick et Dominique Pagès. "Médiation et médiatisation en danse contemporaine : quand la profusion opacifie le sens d'un art sans texte." Quaderni 83 (2013) : 41-58.

Kourlas, Gia. "For Sia, Dance Is Where the Human and the Weird Intersect." The New York Times (2013): 8 .

Mercier-Lefèvre, Betty. “La danse contemporaine et ses rituels.” Corps et Culture 4 (1999). 


\section{Filmographie}

Dans la Peau de John Malkovich. Dir. Spike Jonze. Universal Studios, 1999. DVD.

Her. Dir. Spike Jonze. Warner Bros., 2013. DVD.

Discographie

Sia. "Bird Set Free.” This Is Acting, Monkey Puzzle Records, 2015, piste 1. Spotify, open.spotify.com/album/77jAfTh3KH9K2reMOmTgOh.

Sia. “Chandelier." 1000 Forms of Fear, RCA Records, 2014, piste 1. Spotify, open.spotify.com/album/ 3xFSl9lIRaYXIYkIn3OIl9.

Sia. “Cheap Thrills.” This Is Acting, Monkey Puzzle Records, 2015, piste 6. Spotify, open.spotify.com/album/77jAfTh3KH9K2reMOmTgOh.

Spiegel, Sam, Ape Drums et Assassin. “Mutant Brain." Mutant Brain, Interscope Records, 2016, piste 1. Spotify, open.spotify.com/album/0KrCNabGNzxbl2EUQkW8Tz.

\section{Webographie}

KENZO WORLD. 2018. Visité le 22 avril 2018.

<http://www.kenzoworld.com/fr/>.

Kenzo (2016). KENZO World - The new fragrance. [video] Available at: https://youtu.be/ ABz2m0olmPg [Accessed 22 Apr. 2018].

\section{INDEX}

Keywords : Kenzo, Kenzo World, dance, contemporary dance, marketing, advertising, advertisement

Mots-clés : Kenzo, Kenzo World, danse, danse contemporaine, marketing, publicité Thèmes : Dance

\section{AUTEURS}

\section{CLÉMENT DJAAÏ--MOURGUES}

Étudiant Master Recherche en Études du Monde Anglophone Université Toulouse Jean Jaurès clement.djaai@gmail.com 